\title{
O impacto da Síndrome de Burnout em enfermeiros do setor de urgência e emergência: Uma revisão integrativa da literatura
}

\author{
The impact of Burnout Syndrome on emergency nurses: An integrative review of the literature \\ El impacto del Síndrome de Burnout en enfermeras del sector de urgencia y emergencia: Una \\ revisión integral de la literatura
}

Recebido: 04/12/2020 | Revisado: 14/12/2020 | Aceito: 03/04/2021 | Publicado: 14/04/2021

\author{
Dhayanna Cardoso Lima \\ ORCID: https://orcid.org/0000-0002-0868-2638 \\ Singular Educacional, Brasil \\ E-mail: enfdhayanna10lima@gmail.com \\ Ianny Caroline Mota de Melo \\ ORCID: https://orcid.org/0000-0003-2681-2392 \\ Singular Educacional, Brasil \\ E-mail: ianny-caroline@hotmail.com \\ Clerveson Lima da Costa \\ ORCID: https://orcid.org/0000-0002-7505-3276 \\ Singular Educacional, Brasil \\ E-mail: clervesoncosta@gmail.com \\ Marcilio da Costa Carvalho \\ ORCID: https://orcid.org/0000-0001-6444-4194 \\ Centro Universitário Fametro, Brasil \\ E-mail: marciliocarvalho27@gmail.com
}

\begin{abstract}
Resumo
Objetivo: Discorrer sobre o impacto da Síndrome de Burnout em enfermeiros do setor de urgência e emergência. Metodologia: Trata-se de uma revisão integrativa da literatura, seguindo seis etapas: formação da hipótese ou indagação da pesquisa; amostragem ou busca na literatura; coleta de dados; análise dos estudos incluídos na revisão; interpretação e discussão dos resultados; apresentação da revisão integrativa. Resultados: Os 14 artigos considerados para este estudo foram descritos na tabela, contendo identificação os seguintes itens: título, autor / ano, base de dados, metodologia e resultados. Os resultados identificaram os principais sinais e sintomas de Burnout que acometem o profissional de enfermagem, bem como os fatores que causam impacto na qualidade de vida, e as características do setor de urgência e emergência que influenciam no desencadeamento da síndrome de Burnout. Conclusão: Conclui-se que, o setor de urgência e de um hospital é um local propicio as doenças psicossomáticas aos profissionais que trabalham neste ambiente.
\end{abstract}

Palavras-chave: Burnout; Serviços médicos de emergência; Profissionais de enfermagem.

\begin{abstract}
Objective: To discuss the impact of Burnout Syndrome on emergency nurses. Methodology: This is an integrative review of the literature, following six stages: formation of the hypothesis or inquiry of the research; sampling or search in the literature; data collection; analysis of the studies included in the review; interpretation and discussion of the results; presentation of the integrative review. Results: The 15 articles considered for this study were described in the table, containing identification of the following items: title, author / year, database, methodology and results. The results identified the main signs and symptoms of Burnout that affect the nursing professional, as well as the factors that impact on quality of life, and the characteristics of the emergency department that influence the onset of Burnout syndrome. Conclusion: It is concluded that, the emergency department and a hospital is a place propitious psychosomatic diseases to professionals working in this environment.
\end{abstract}

Keywords: Burnout; Emergency medical services; Nursing professionals.

\section{Resumen}

Objetivo: Discurrir sobre el impacto del Síndrome de Burnout en enfermeras del sector de urgencia y emergencia. Metodología: Se trata de una revisión integrativa de la literatura, siguiendo seis etapas: formación de la hipótesis o indagación de la investigación; muestreo o búsqueda en la literatura; recogida de datos; análisis de los estudios incluidos en la revisión; interpretación y discusión de los resultados; presentación de la revisión integrativa. Resultados: Los 15 artículos considerados para este estudio fueron descritos en la tabla, conteniendo identificación los siguientes ítems: título, autor / año, base de datos, metodología y resultados. Los resultados identificaron los 
principales signos y síntomas de Burnout que afectan al profesional de enfermería, así como los factores que influyen en la calidad de vida, y las características del sector de urgencia y emergencia que influyen en el desencadenamiento del síndrome de Burnout. Conclusión: Se concluye que el sector de urgencia y de un hospital es un lugar propicio a las enfermedades psicosomáticas a los profesionales que trabajan en este ambiente.

Palabras clave: Burnout; Servicios médicos de emergencia; Profesionales de enfermería.

\section{Introdução}

O trabalho executado no setor de urgência e emergência tem características próprias que podem contribuir para a Síndrome de Burnout - SB. Com isso, é necessário ter um amplo entendimento do que está acontecendo nessa área. Verificase que as atividades realizadas, a falta de tempo, o grande número de pacientes e a necessidade de agilidade nas ações de enfermagem tornam os enfermeiros do departamento mais vulneráveis a questões relacionadas ao estresse (Kolhs \& Olschowsy \& Barreta, 2017).

A Síndrome de Burnout - SB, é definida pela dimensão da exaustão emocional e é confirmado pelo sentimento de fadiga, cansaço físico e emocional e despersonalização, ou seja, o indivíduo está longe das relações interpessoais, e devido à redução da realização profissional, o indivíduo proporciona um sentimento negativo para si mesmo. A síndrome é insidiosa e está relacionada a profissionais que costumam lidar com pessoas e estressores no ambiente de trabalho (Lima \& Farah \& Bustamante-Teixeira, 2018).

É citado que os sintomas deste esgotamento podem ser mental, físico, psicológico e comportamental, e geralmente têm um impacto negativo nos níveis pessoal, profissional e social. Em geral, os indivíduos estão exaustos mental e fisicamente, geralmente irritados, ansiosos ou tristes. Além disso, a frustração emocional não apenas leva ao abuso de álcool e drogas, mas também causam úlceras, insônia, dores de cabeça e pressão alta, o que intensifica os problemas familiares e os conflitos sociais. As consequências para as instituições incluem alto absenteísmo, acidentes de trabalho, baixa por doença, qualidade de vida no trabalho reduzida e aumento do conflito interpessoal (Cardoso et al., 2018).

A equipe de enfermagem está inserida em uma sociedade capitalista onde novos ideais surgem a cada dia, e preocupase em cumprir tarefas e exigências. Por esse motivo, muitas vezes estabelecem contatos com duas ou mais instituições para a realização de atividades laborais. Além da fiscalização dos serviços de saúde, essas atividades também podem gerar sobrecarga de trabalho e estresse, que afetam diretamente o comportamento biopsicossocial e a qualidade de vida dos profissionais (Amador et al.,2013).

A SB causa impacto direto nos cuidados prestados aos pacientes e na relação entre profissionais e colegas, além de afetar a qualidade de vida e a saúde dos funcionários fora do ambiente de laboral, porque se sentem deprimidos e exaustos física e mentalmente, o que pode interferir e reduzir suas funções regulares. Pode-se supor até mesmo que o Burnout seja uma questão de saúde pública, haja vista que um profissional cansado e adoecido mentalmente não possui condições para cuidar de forma efetiva de um doente (Oliveira \& Lima \& Vilela, 2017).

É necessário um bom gerenciamento de escala e dimensionamento de pessoal. No entanto, esse tipo de atribuição às vezes consome a saúde ocupacional dos profissionais, portanto, devido às tarefas excessivas atribuídas aos profissionais, às vezes não basta caracterizar o ambiente de trabalho, diferenciando o ambiente de trabalho. Diante dessa situação, é compreensível que os trabalhadores se sintam fatigados devido ao serviço excessivo, que não apenas causa fadiga física, mas também fadiga emocional, que afeta sua saúde. (Paiva et al., 2019).

A realidade vivida nos setores de emergência e emergência significa que os profissionais de saúde estão sempre expostos a riscos físicos e psicológicos, agindo sob intensa pressão todos os dias e precisam saber como lidar com o risco iminente de morte. Isso leva facilmente aos sintomas e sinais da síndrome de Burnout (Angelim \& Rocha, 2016).

Este trabalho justifica-se pela necessidade em discutir os impactos da SB na qualidade de vida dos enfermeiros, 
trazendo benefícios diversos para a pratica da assistência aos pacientes, para as comunidades, e para as equipes de atendimento, pois produz informações acerca dos sinais e sintomas mais comuns. Além disso, o conhecimento gerado ainda pode ser utilizado na formação acadêmica dos futuros profissionais de enfermagem.

Portanto, o objetivo deste estudo é discorrer sobre o impacto da Síndrome de Burnout em enfermeiros do setor de urgência e emergência de acordo com a literatura.

\section{Metodologia}

Trata-se de uma revisão integrativa da literatura, que busca discorrer sobre o impacto da Síndrome de Burnout em enfermeiros do setor de urgência e emergência. A pesquisa seguiu seis etapas: formação da hipótese ou indagação da pesquisa; amostragem ou busca na literatura; coleta de dados; análise dos estudos incluídos na revisão; interpretação e discussão dos resultados; apresentação da revisão integrativa (Ganong, 2011).

No primeiro passo foi elaborada a seguinte questão de pesquisa para guiar o estudo: Qual o impacto da Síndrome de Burnout em enfermeiros do setor de urgência e emergência? Para a busca dos resultados desta pesquisa, serão utilizados os seguintes descritores em Ciências da Saúde (DeCS): Burnout, serviços médicos de emergência, profissionais de enfermagem.

A pesquisa bibliográfica foi consumada por meio de consulta nas bases de dados SciELO - Scientific Electronic Library, LILACS- Literatura Latino-americana, BDENF - Banco de Dados em Enfermagem. Foi definitiva esta estratégia de pesquisa, conveniente à triagem cuidadosa e objetividade de apuração das informações manifestadas. Tal busca ocorreu nos meses de maio e junho de 2020.

Fazendo parte apenas artigos primários, com texto completo, disponível on-line na íntegra, com acesso livre. A escolha por este tipo de publicação deve-se à maior acessibilidade as produções cientificas. Para o registro das informações de cada artigo, as informações extraídas foram: título, autor, ano, base de dados, metodologia e resultados. Os dados obtidos através dos instrumentos foram organizados e categorizados em uma planilha eletrônica do Microsoft Office Word para análise qualitativa.

Para a análise da coleta de dados, foi realizada a leitura uma leitura flutuante para abordagem e confirmação das ideias, que utilizou análise de conteúdo temática e sistematização dos conteúdos científicos selecionados.

Os critérios de inclusão delimitados para pré-seleção dos estudos instituídos foram: contemplar o objetivo proposto; ter sido publicado no período de 2015 a 2020; estar no idioma português e disponível eletronicamente na íntegra. Foram excluídos os editoriais, cartas ao editor, trabalhos publicados em anais de eventos, textos incompletos, em outros idiomas e artigos repetidos.

O total geral de artigos encontrados nestas bases utilizando os descritores foi de 406 artigos, sendo na SCIELO 250 artigos, e LILACS 96 artigos e na BDENF foram encontrados 60 artigos. Conforme o cruzamento dos descritores: Serviços médicos de emergência e Burnout na LILACS, encontramos 28 artigos, na SCIELO 5 artigos e na BDENF 17 artigos. Utilizando os descritores profissionais de enfermagem e Burnout, foram encontrados na LILACS 222 artigos publicados, na SCIELO 91 artigos, e na BDENF 43 artigos.

No registro após se eliminarem os artigos duplicados restaram 259 artigos, sendo excluídos 197 artigos. Sendo avaliados os artigos completos conforme os critérios de elegibilidade apenas 101 artigos foram de acordo com esses critérios.

Correspondendo aos objetivos específicos da temática pré-estabelecida foram incluídos nesta síntese qualitativa 14 artigos ao total. As informações extraídas de cada artigo, foram: título, autor, ano, base de dados, metodologia e resultados. Os dados foram obtidos através da pesquisa na base de dados que sendo organizados e categorizados em uma planilha eletrônica do Microsoft Office Word para análise qualitativa.

Para a execução deste estudo não foi necessário consentimento dos protagonistas da pesquisa, estando de acordo com 
a Resolução 466/2012, do Conselho Nacional de Saúde, uma vez que o estudo se trata de uma revisão integrativa da literatura. Porém, enfatiza-se que ao longo do progresso deste trabalho, respeita-se a autoria das informações utilizadas no suporte científico, como uma forma de reconhecer a originalidade da produção intelectual dos pesquisadores.

\section{Resultados e discussões}

\subsection{Perfil da amostra}

Foram identificados 14 artigos dos quais todos pertenciam a diferentes bases de dados, mencionados na síntese abaixo. Após a aplicação dos critérios de elegibilidade e inelegibilidade a amostra final do estudo foi composta por 14 artigos. Com isso, para melhor discutir os resultados elegeram-se duas categorias.

Quadro 1 - Síntese dos estudos incluídos na revisão.

\begin{tabular}{|c|c|c|c|c|}
\hline Título & Autor/ Ano & $\begin{array}{l}\text { Base de } \\
\text { dados }\end{array}$ & Metodologia & Resultados \\
\hline $\begin{array}{l}\text { Processo de trabalho e } \\
\text { seu impacto nos } \\
\text { profissionais } r \text { de } \\
\text { enfermagem em } \\
\text { de serviço } \\
\text { de saúde mental. }\end{array}$ & $\begin{array}{l}\text { SOUZA et al., } \\
2015\end{array}$ & SCIELO & $\begin{array}{l}\text { Estudo } \\
\text { transversal. }\end{array}$ & $\begin{array}{l}\text { Os profissionais de Enfermagem seu processo } \\
\text { de trabalho, estiveram expostos a todas as } \\
\text { cargas de desgaste, sendo o desgaste psíquico } \\
\text { mais intenso que o físico, o qual refletiu na } \\
\text { insatisfação com a atividade laboral e na saúde } \\
\text { física dos trabalhadores. }\end{array}$ \\
\hline $\begin{array}{l}\text { Risco de adoecimento } \\
\text { dos profissionais de } \\
\text { enfermagem no trabalho } \\
\text { em atendimento móvel } \\
\text { de urgência. }\end{array}$ & $\begin{array}{l}\text { WORM et al., } \\
2016\end{array}$ & SCIELO & $\begin{array}{l}\text { Estudo } \\
\text { transversal. }\end{array}$ & $\begin{array}{l}\text { Os resultados mostram que a equipe de } \\
\text { enfermagem do Serviço de Atendimento Móvel } \\
\text { de Urgência apresenta riscos de adoecimento, } \\
\text { principalmente relacionado ao fator físico e } \\
\text { psicológico. }\end{array}$ \\
\hline $\begin{array}{l}\text { A enfermagem na } \\
\text { urgência e emergência: } \\
\text { entre o prazer e o } \\
\text { sofrimento }\end{array}$ & $\begin{array}{l}\text { KOLHS et al., } \\
2017\end{array}$ & BDENF & $\begin{array}{l}\text { Estudo } \\
\text { qualitativo, } \\
\text { descritivo, } \\
\text { estudo de caso. }\end{array}$ & $\begin{array}{l}\text { Mudanças de condutas podem permitir uma } \\
\text { melhor forma de lidar e transformar os fatores } \\
\text { geradores de sofrimento e potencializar os } \\
\text { sentimentos de prazer. }\end{array}$ \\
\hline $\begin{array}{l}\text { Síndrome de burnout em } \\
\text { profissionais do serviço } \\
\text { de atendimento móvel de } \\
\text { urgência. }\end{array}$ & LUZ et al., 2017 & BDENF & $\begin{array}{l}\text { Estudo } \\
\text { descritivo } \\
\text { transversal }\end{array}$ & $\begin{array}{l}\text { Para a equipe como um todo, houve escores } \\
\text { altos para Desgaste Emocional }(28,1 \%) \text {, } \\
\text { Despersonalização }(21,9 \%) \text { e Incompetência } \\
\text { Profissional }(28,1 \%) \text { (escore reverso). Na } \\
\text { equipe médica, o Desgaste Emocional e } \\
\text { Despersonalização foram maiores quando } \\
\text { comparado a equipe de enfermagem; e em } \\
\text { Incompetência Profissional os escores foram } \\
\text { mais elevados para os técnicos de enfermagem. }\end{array}$ \\
\hline $\begin{array}{l}\text { Impacto do ambiente de } \\
\text { cuidados críticos no } \\
\text { Burnout, percepção da } \\
\text { qualidade do cuidado e } \\
\text { atitude de segurança da } \\
\text { equipe de enfermagem. }\end{array}$ & $\begin{array}{l}\text { GUIRARDELLO } \\
\text { et al., } 2017\end{array}$ & SCIELO & $\begin{array}{l}\text { Estudo } \\
\text { transversal }\end{array}$ & $\begin{array}{l}\text { Os profissionais que perceberam maior } \\
\text { autonomia, boas relações com a equipe médica } \\
\text { e melhor controle sobre o ambiente de trabalho, } \\
\text { apresentaram menores níveis de Burnout, } \\
\text { avaliaram como boa a qualidade do cuidado e } \\
\text { relataram uma percepção positiva da atitude de } \\
\text { segurança para o domínio satisfação no } \\
\text { trabalho. }\end{array}$ \\
\hline $\begin{array}{l}\text { Estresse ocupacional em } \\
\text { profissionais de } \\
\text { enfermagem }\end{array}$ & $\begin{array}{l}\text { LLAPA- } \\
\text { RODRIGUEZ } \\
\text { BORGES et al., } \\
2018\end{array}$ & SCIELO & $\begin{array}{l}\text { Pesquisa } \\
\text { descritiva } \\
\text { correlacional }\end{array}$ & $\begin{array}{l}\text { A enfermagem se revelou como uma profissão } \\
\text { de risco para o estresse ocupacional, sofrendo } \\
\text { diversas influências dos fatores analisados, } \\
\text { relacionados à organização e ao processo de } \\
\text { trabalho. }\end{array}$ \\
\hline
\end{tabular}




\begin{tabular}{|c|c|c|c|c|}
\hline $\begin{array}{l}\text { Fadiga por compaixão } \\
\text { em enfermeiros de } \\
\text { urgência e emergência } \\
\text { hospitalar de adultos. }\end{array}$ & $\begin{array}{l}\text { BORGES et al., } \\
2019\end{array}$ & LILACS & $\begin{array}{l}\text { Estudo } \\
\text { quantitativo, } \\
\text { descritivo e } \\
\text { transversal. }\end{array}$ & $\begin{array}{l}\text { Existe fadiga por compaixão expressa na } \\
\text { grande percentagem de enfermeiros com } \\
\text { elevados níveis de Burnout e de estresse } \\
\text { traumático secundário. A fadiga depende de } \\
\text { fatores individuais como idade, sexo, } \\
\text { experiência profissional e atividades de lazer. }\end{array}$ \\
\hline $\begin{array}{l}\text { Fatores relacionados à } \\
\text { probabilidade de sofrer } \\
\text { problemas de saúde } \\
\text { mental em profissionais } \\
\text { de emergência }\end{array}$ & CRUZ et al., 2019 & LILACS & $\begin{array}{l}\text { Estudo } \\
\text { descritivo } \\
\text { transversal }\end{array}$ & $\begin{array}{l}\text { O aumento da experiência profissional esteve } \\
\text { associado à maior disfunção social do pessoal } \\
\text { de saúde e o aumento do número de pacientes } \\
\text { foi relacionado à sintomatologia depressiva } \\
\text { entre os profissionais de saúde. }\end{array}$ \\
\hline $\begin{array}{lr}\text { Características } & \text { do } \\
\text { trabalho e } & \text { estresse } \\
\text { ocupacional } & \text { entre } \\
\text { Enfermeiros hospitalares }\end{array}$ & $\begin{array}{l}\text { ROCHA et al., } \\
2019\end{array}$ & LILACS & $\begin{array}{l}\text { Estudo } \\
\text { transversal }\end{array}$ & $\begin{array}{l}\text { Houve prevalência de trabalhadores que } \\
\text { possuem alta demanda no trabalho e alto } \\
\text { controle, o que caracterizou maior proporção de } \\
\text { trabalho ativo. Apesar de estarem expostos à } \\
\text { alta demanda de trabalho, os enfermeiros } \\
\text { referiram alto controle e suporte social ao labor, } \\
\text { o que determinou maior concentração do } \\
\text { trabalho ativo na amostra, logo, pouco estresse. }\end{array}$ \\
\hline $\begin{array}{l}\text { O esgotamento físico do } \\
\text { enfermeiro do setor de } \\
\text { urgência e emergência: } \\
\text { revisão integrativa. }\end{array}$ & $\begin{array}{l}\text { OLIVEIRA et al., } \\
2019\end{array}$ & BDENF & $\begin{array}{l}\text { Revisão } \\
\text { integrativa. }\end{array}$ & $\begin{array}{l}\text { É mostrada a necessidade de intervenções, as } \\
\text { quais reduzam a prevalência de Burnout entre } \\
\text { os profissionais da saúde, melhorando o bem- } \\
\text { estar físico e psicológico e potencializando o } \\
\text { serviço com qualidade. }\end{array}$ \\
\hline $\begin{array}{l}\text { Trabalho em turnos de } \\
\text { profissionais } \\
\text { enfermagem e a pressão } \\
\text { arterial, Burnout e } \\
\text { transtornos mentais } \\
\text { comuns. }\end{array}$ & $\begin{array}{l}\text { NASCIMENTO } \\
\text { et al., } 2019\end{array}$ & SCIELO & $\begin{array}{l}\text { Estudo } \\
\text { transversal. }\end{array}$ & $\begin{array}{l}\text { Os trabalhadores de enfermagem que atuavam } \\
\text { em turnos apresentaram maior prevalência de } \\
\text { fatores negativos relacionados ao trabalho, } \\
\text { hábitos e estilos de vida inadequados, além de } \\
\text { alteração da pressão no período de sono, } \\
\text { quando comparados aos trabalhadores que não } \\
\text { estavam expostos a essa escala de trabalho. }\end{array}$ \\
\hline $\begin{array}{l}\text { Estresse Ocupacional: } \\
\text { Exposição da Equipe de } \\
\text { Enfermagem de uma } \\
\text { Unidade de Emergência }\end{array}$ & $\begin{array}{l}\text { SANTOS et al., } \\
2019\end{array}$ & BDENF & $\begin{array}{l}\text { Estudo } \\
\text { qualitativo }\end{array}$ & $\begin{array}{l}\text { Os profissionais da equipe de enfermagem da } \\
\text { Emergência estão expostos a fatores de riscos } \\
\text { psicológicos, inclusive ao estresse ocupacional, } \\
\text { devido à sobrecarga de trabalho, à demanda } \\
\text { maior do que as condições assistenciais da } \\
\text { equipe e ao número insuficiente de } \\
\text { profissionais da enfermagem no setor. }\end{array}$ \\
\hline $\begin{array}{l}\text { Síndrome de Burnout em } \\
\text { profissionais de } \\
\text { enfermagem }\end{array}$ & $\begin{array}{l}\text { COSTA et al., } \\
2020\end{array}$ & SCIELO & $\begin{array}{l}\text { Estudo } \\
\text { quantitativo, } \\
\text { descritivo, } \\
\text { transversal. }\end{array}$ & $\begin{array}{l}\text { Houve predominância do nível moderado nas } \\
\text { três dimensões do Burnout, resultado que se } \\
\text { mostra preocupante, uma vez que, como } \\
\text { defendido por Betty Neuman em sua teoria, a } \\
\text { estabilidade do sistema e a necessidade deste } \\
\text { ser mantido em equilíbrio são essenciais para } \\
\text { evitar o adoecimento psíquico, inclusive, no } \\
\text { ambiente laboral. }\end{array}$ \\
\hline $\begin{array}{l}\text { Síndrome de Burnout em } \\
\text { profissionais de } \\
\text { enfermagem de pronto- } \\
\text { socorro }\end{array}$ & PIRES et al., 2020 & & $\begin{array}{l}\text { Estudo } \\
\text { quantitativo, } \\
\text { descritivo, } \\
\text { transversal. }\end{array}$ & $\begin{array}{l}\text { Houve associação significativa entre as } \\
\text { variáveis sociodemográficas com a Síndrome } \\
\text { de Burnout. Sugere-se um plano de prevenção à } \\
\text { saúde do trabalhador individualizado. }\end{array}$ \\
\hline
\end{tabular}

Fonte: Autores. 


\section{Sinais e sintomas de Burnout que acometem o profissional de enfermagem do setor de urgência e emergência.}

Com o passar dos anos, os serviços prestados pela equipe de enfermagem desencadeiam sinais e sintomas de doenças ocupacionais. O que afeta suas habilidades de trabalho e causa esgotamento emocional, irritabilidade, síndrome da fadiga crônica, distúrbios do sono, depressão, síndrome da fadiga profissional e estresse ocupacional (Llapa-rodriguez et al., 2018).

De acordo com estudos, a maioria dos enfermeiros classificados com o estresse relata sintomas psicológicos e físicos. Em um ambiente insalubre, a qualidade do sono ao trabalhar em más condições físicas limita seu trabalho e causam exaustão emocional. A fadiga emocional relacionada ao trabalho realizado pelos profissionais está significativamente relacionada à ocorrência de estresse (Carvalho et al., 2020).

A fadiga da compaixão é referida como a causa por alto desgaste e alto estresse traumático secundário, afetando a personalidade, impactando a experiência profissional e qualidade de vida pessoal em nível individual. Devido à demanda e exposição frequente a traumas, o trabalho de enfermagem em emergências e emergências facilita para os enfermeiros internalizarem a dor dos pacientes, aumentando assim a fadiga por compaixão, favorecendo o surgimento de SB (Borges et al., 2019).

Os autores mencionam que a SB tem como definição o esgotamento da própria energia ou dos recursos emocionais resultantes da energia excessiva que um indivíduo dedica ao trabalho, tornando impossível para ele dar a si mesmo a sensação de estar sobrecarregado por tarefas e pressão. Nesse caso, o profissional sente que está no limite (Costa et al., 2020).

Os sintomas físicos mais comuns apresentados pelos profissionais da enfermagem são: Cansaço, fadiga, dor de cabeça ou mal-estar geral. Os sintomas psicológicos, conforme apresenta os autores são: Transtornos de ansiedade (dificuldade em adormecer, acordar com frequência, irritabilidade ou nervosismo), disfunção social (incapacidade de tomar decisões ou desenvolvimento do trabalho organizado, levando à deterioração das funções diárias) e depressão (sintomas relacionados ao humor, incluindo ideação suicida) (Cruz et al., 2019).

Quanto aos profissionais que atuam no Serviço de Atendimento Móvel de Urgência - SAMU, também é relatado em estudos a insatisfação com a organização do local de trabalho como um fator predisponente a SB. Sobre os indicadores de prazer e dor no trabalho, destacam fadiga profissional e falta de reconhecimento, com ênfase em "desvalorização", "falta de reconhecimento dos meus esforços", e "insatisfação", indicando que, neste caso, a exaustão e a falta de reconhecimento da equipe são um problema que afeta a saúde física e mental desses trabalhadores (Worm et al., 2016).

Reforça-se a sobrecarga como a causa da insatisfação com o trabalho e a profissão, o que pode fazer com que os profissionais de enfermagem mudem de emprego. Há evidências de que isso acontece com mais frequência entre os enfermeiros que trabalham em ambiente hospitalar. Em comparação com os enfermeiros que trabalham em departamentos de serviços comunitários, os profissionais que trabalham em hospitais apresentam escores mais altos de despersonalização. Os conflitos e violência no local de trabalho podem aumentar a carga psicológica dos profissionais de enfermagem (Souza et al., 2015).

\section{Fatores causadores de impacto na qualidade de vida dos profissionais de enfermagem que atuam na urgência e emergência.}

Indivíduos ao assumirem muitos papéis na vida usualmente enfrentam grande peso, tanto na vida familiar como no trabalho, gerando conflitos em relação ao seu ambiente laboral. Condições, principalmente, em decorrência, do exercício laboral com longas jornadas de trabalho e prestação de cuidados a pessoas em situação de vulnerabilidade biológica e psicológica, com sofrimentos psíquicos e estados terminais (Llapa-rodriguez et al., 2018). 
A própria rotina de trabalho na emergência é um fator capaz de afetar o quadro emocional do trabalhador, e a exposição por um tempo longo ao estresse ocupacional pode desencadear o desenvolvimento da síndrome do desgaste emocional, que confere ao portador grande exaustão emociona (Carvalho et al., 2020).

Ambientes favoráveis à prática profissional da equipe de enfermagem podem resultar em menores níveis de exaustão emocional, melhor qualidade do cuidado e uma percepção positiva sobre atitudes de segurança (Guirardello et al., 2017).

A jornada de trabalho densa e as múltiplas funções também foram referidas como cargas física às quais os profissionais de Enfermagem estiveram expostos, constituindo-se em sobrecargas de trabalho e geradoras de impactos negativos na saúde do trabalhador (Souza et al., 2015).

Os trabalhadores de enfermagem que atuam em turnos comparando aos trabalhadores que não estão expostos a essa escala de trabalho, além das mudanças no estresse durante o sono, a prevalência de fatores negativos relacionados ao trabalho, hábitos inadequados e estilo de vida também é maior. De acordo com os resultados da investigação, é necessário monitorar e monitorar os profissionais que apresentam fatores de risco e todos os profissionais que podem trabalhar em turnos (Nascimento et al., 2019).

A enfermagem passa diariamente por sofrimento dentro do seu local de trabalho, e um dos principais motivos deste sofrimento é a morte, a qual gera sentimento de tristeza, fazendo com que o profissional busque auxílio, refúgio em vários lugares, no entanto o sentimento de tristeza permanece impregnado em si (Worm et al., 2016).

Vale ressaltar que a Síndrome de Burnout pode ser causada por desgaste em longo prazo durante o tempo de atuação. Quanto maior o tempo de serviço em uma determinada área, maior o nível de estresse e, portanto, há uma correlação significativamente alta entre a pressão na área e o tempo de serviço (Luz et al., 2017).

\section{Características do setor de urgência e emergência que influenciam no desencadeamento da síndrome de Burnout.}

O setor de urgência e emergência é caracterizado por alguns profissionais como sendo um setor entre "o prazer e o sofrimento". O prazer de um serviço esta na assistência de alta qualidade, como também em estar satisfeito com seu desempenho, além de ser capaz de ajudar e ser útil para salvar vidas e proporcionar melhorias aos pacientes. Já o sofrimento é caracterizado por situações frustrantes no ambiente de trabalho, como impotência, desânimo, insatisfação, tristeza, e outros sentimentos que causam exaustão física e mental no trabalho, prejudicando o desempenho do profissional, o que pode fazê-lo se sentir deprimido e doente (Kolhs et al., 2017).

Nas enfermarias e postos de emergência, o número de profissionais da equipe de enfermagem, geralmente é insuficiente para atender às necessidades identificadas, o que reflete que a qualidade da assistência e o estado de saúde dos enfermeiros e técnicos de enfermagem passam a se comprometer, estando ocupados na realização de diversas atividades compostas por poucos profissionais (Santos et al., 2019).

O trabalho na emergência é realizado com o apoio de muitos dispositivos necessários para manter a vida e estabilizar os pacientes, que podem causar estresse, ansiedade e dor aos profissionais de saúde, pois podem responder a situações que exigem agilidade, raciocínio rápido e tecnologia aprimorada. Nesse caso, essas pessoas precisam suportar a pressão profissional e a pressão no trabalho em longo prazo e, como resultado, sofrem da SB (Worm et., 2016).

Nas atividades desempenhadas pelos profissionais que atuam no SAMU, são citadas respostas positivas ao estresse, os aspectos mais afetados são a sobrecarga mental e física, caso não sejam evitadas, essas condições prejudicam a saúde, a produtividade e a organização do trabalho dos trabalhadores. Serviços, especialmente assistência ao publico (Adriano et al., 2017).

Os trabalhos dos serviços hospitalares representam um alto risco de doença física e mental dos trabalhadores. A julgar pelo processo profissional (incluindo assistência, gestão, administração, pesquisa, ensino e participação política), supõe-se que 
ele tenha experimentado condições de alta demanda / exigência. Portanto, é importante que, além das associações profissionais, existam ações que possam amenizar essa situação e que exijam planejamento constante por parte do gerente de atenção e saúde (Rocha et al., 2019).

Os enfermeiros emergêncistas precisam ser capazes de tomar decisões rapidamente, para avaliar efetivamente os pacientes. Esta dinâmica de atendimento requer que esses profissionais sejam agentes direcionados, enfatizando que pacientes com condições graves não podem suportar longas esperas e não causará má conduta devido à assistência (Oliveira et al., 2019).

\section{Considerações Finais}

Dada à relevância da temática abrangida, conclui-se que, os profissionais da enfermagem, com destaque aos enfermeiros, são bastante afetados por esta síndrome que é bastante perigosa, a Síndrome de Burnout. O setor de urgência e de um hospital é um local propicio as doenças psicossomáticas por ser um ambiente que exige habilidade, raciocínio rápido e conhecimento de tecnologias.

Considerando as manifestações clinicas da SB causadas por sobrecarga de trabalho, ambiente insalubre, alta demanda, dimensionamento de pessoal ineficaz entre outros fatores que o setor impõe, é bastante compreensível, as consequências serem calamitosas.

Percebe-se, diante disso, a necessidade da realização de novas pesquisas acerca da Síndrome de Burnout em profissionais que atuam na urgência e emergência a fim de conscientizar os indivíduos sobre os sintomas causados pela doença mental, ajudando assim a melhorar a qualidade de vida dos profissionais de enfermagem para alcançar uma enfermagem qualificada e eficaz.

\section{Referências}

Amador, J. C. et al (2013). Síndrome de Burnout em profissionais de enfermagem. Revista OMNIA Saúde,. 10.

Angelim, R., \& Rocha, G. (2016). Scientific production about the working conditions of nursing in emergency and urgent services. Revista de Pesquisa: Cuidado é Fundamental Online, 8(1), 3845-3859. http://dx.doi.org/10.9789/2175-5361.2016.v8i1.3845-3859

Bardin, L. (2011). Análise de conteúdo. Tradução de Luis Antero reto e augusto pinheiro. Livraria Martins Fontes.

Borges, E. M. N., Fonseca, C. I. N. da S., Baptista, P. C. P., Queirós, C. M. L., Baldonedo-Mosteiro, M., \& Mosteiro-Diaz, M. P. (2019). Fadiga por compaixão em enfermeiros de urgência e emergência hospitalar de adultos. Revista Latino-Americana de Enfermagem, 27, e3175. https://doi.org/10.1590/1518-8345.2973.3175

Cardoso, H. F., Baptista, M. N., Sousa, D. F. A. \& Goulart Júnior, E. (2017). Síndrome de burnout: análise da literatura nacional entre 2006 e 2015. Revista Psicologia Organizações e Trabalho, 17(2), 121-128. https://dx.doi.org/10.17652/rpot/2017.2.12796

Carvalho, A. E. L., Frazão, I. da S., Silva, D. M. R. da, Andrade, M. S., Vasconcelos, S. C., \& Aquino, J. M. (2020). Estresse dos profissionais de enfermagem atuantes no atendimento pré-hospitalar. Revista Brasileira de Enfermagem, 73(2), e20180660. https://doi.org/10.1590/0034-7167-2018-0660

Cruz, S. P., Cruz, J. C., Cabrera, J. H., \& Abellán, M. V. (2019). Fatores relacionados à probabilidade de sofrer problemas de saúde mental em profissionais de emergência. Revista Latino-Americana de Enfermagem, 27, e3144. https://doi.org/10.1590/1518-8345.3079-3144

Guirardello, E. de B. (2017). Impact of critical care environment on burnout, perceived quality of care and safety attitude of the nursing te am. Revista LatinoAmericana de Enfermagem, 25, e2884. https://doi.org/10.1590/1518-8345.1472.2884

Kolhs, M., Olschowsky, A., Barreta, N., Schimerfening, J., Vargas, R., \& Busnello, G. (2017). A enfermagem na urgência e emergência: entre o prazer e o sofrimento Nursing in urgency and emergency: between the pleasure and suffering. Revista de Pesquisa: Cuidado é Fundamental Online, 9(2), 422-431. http://dx.doi.org/10.9789/2175-5361.2017.v9i2.422-431

Llapa-Rodriguez, E., Oliveira, J., Lopes Neto, D., Gois, C., Campos, M., \& Mattos, M. (2018). Estresse ocupacional em profissionais de enfermagem Revista Enfermagem UERJ, 26, e19404. https://doi.org/10.12957/reuerj.2018.19404

Lima, A. de S., Farah, B. F., \& Bustamante-Teixeira, M. T. (2018). Análise da prevalência da síndrome de burnout em profissionais da atenção primária em saúde. Trabalho, Educação e Saúde, 16(1), 283-304. https://doi.org/10.1590/1981-7746-sol00099 
Research, Society and Development, v. 10, n. 4, e36110410907, 2021

(CC BY 4.0) | ISSN 2525-3409 | DOI: http://dx.doi.org/10.33448/rsd-v10i4.10907

Luz, L. M. et al. Síndrome de burnout em profissionais do serviço de atendimento móvel de urgência Burnout Syndrome in urgency mobile service professionals. Revista de Pesquisa: Cuidado é Fundamental Online, 9(1), 238, Universidade Federal do Estado do Rio de Janeiro UNIRIO. http://dx.doi.org/10.9789/2175-5361.2017.v9i1.238-246.

Nascimento, J. O. V., Santos, J., Meira, K. Cardoso, P., Angela M. G., \& Souza-Talarico, J. N. (2019). Turnos de trabalho dos profissionais de enfermagem e hipertensão, burnout e transtornos mentais comuns. Revista da Escola de Enfermagem da USP, 53, https://dx.doi.org/10.1590/s1980-220x2018002103443

Rocha, R., Valim, M., de Oliveira, J., \& Ribeiro, A. (2020). Características do trabalho e estresse ocupacional entre enfermeiros hospitalares. Enfermagem em Foco, 10(5). https://doi.org/10.21675/2357-707X.2019.v10.n5.2581

Santos J. N. M. O. et al., Estresse Ocupacional: Exposição da Equipe de Enfermagem de uma Unidade de Emergência. Rev Fund Care Online. $455-463$. http://dx.doi. org/10.9789/2175-5361.2019.v11i2.455-463

Souza, I. A. S. et al (2015). Processo de trabalho e seu impacto nos profissionais de enfermagem em serviço de saúde mental. Acta paul. enferm., 28(5), 447453. https://doi.org/10.1590/1982-0194201500075.

Worm, F. A. et al. (2016). Risco de adoecimento dos profissionais de enfermagem no trabalho em atendimento móvel de urgência. Rev Cuid, Bucaramanga, 7(2), 1279-1287. http://dx.doi.org/10.15649/cuidarte.v7i2.329. 